Open Access

\title{
Correlation of PET-CT nodal SUVmax with p16 positivity in oropharyngeal squamous cell carcinoma
}

\author{
Jessica Clark' , Caroline C. Jeffery ${ }^{1}$, Han Zhang ${ }^{1}$, Tim Cooper ${ }^{1}$, Daniel A. O'Connell', Jeffrey Harris ${ }^{1}$, Hadi Seikaly ${ }^{1}$
} and Vincent L. Biron ${ }^{1,2^{*}}$

\begin{abstract}
Background: The incidence of human papillomavirus (HPV)-related oropharyngeal squamous cell carcinoma (OPSCC) has been rising in recent years. Given the clinical impact of HPV/p16 positivity in OPSCC, identifying surrogate markers of this disease early in the diagnostic work-up of these patients could improve patient care.
\end{abstract}

Methods: Demographic, pathologic, staging and PET-CT data from patients diagnosed with OPSCC from 2009-2014 were obtained from a prospectively collected provincial cancer registry. Tumor HPV/p16 status was correlated to the maximum standard uptake value (SUVmax) of the primary tumor and cervical nodes. Comparisons of means and multinomial regression models were used to determine associations between p16 status and SUVmax. A diagnostic odds ratio was calculated using a cut off value for predicting HPV/p16 positivity based on nodal SUVmax.

Results: PET-CT and HPV/p16 data was obtained for 65 patients treated surgically for OPSCC. Significantly higher nodal SUVmax was associated with HPV/p16 positive nodes (SUVmax 10.8 vs 7.9). No significant differences were seen between HPV/p16 positive vs negative primary tumor SUVmax (10.3 vs 13.7). In combination with other clinical parameters, higher nodal SUVmax was highly correlated with HPV/p16 positivity.

Conclusion: Elevated nodal SUVmax is a significant predictor of HPV/p16 positive disease.

Keywords: Oropharyngeal cancer, Positron emission tomography, Standard uptake value, p16, Human papillomavirus

\section{Introduction}

Oropharyngeal squamous cell carcinoma (OPSCC) is an aggressive malignancy with a rising incidence worldwide [1-3]. Oncogenic human papillomavirus (HPV) infection, tobacco and alcohol are well established etiological factors for OPSCC $[4,5]$. A large body of evidence from recent years has demonstrated that HPV positive and negative OPSCC are distinct from clinical, pathological and molecular perspectives [4, 6-12]. Most importantly; patients with HPV-related OPSCC, generally pathologically identified by p16 positivity, have favorable survival outcomes following both surgical and non-surgical

\footnotetext{
* Correspondence: vbiron@ualberta.ca

${ }^{1}$ Faculty of Medicine and Dentistry, Department of Surgery, Division of Otolaryngology-Head and Neck Surgery, University of Alberta, Edmonton, Alberta, Canada

${ }^{2}$ University of Alberta, Otolaryngology-Head and Neck Surgery, 1E4, Walter Mackenzie Centre, University of Alberta Hospital, 8440-112St, Edmonton,
} Alberta T6G 2B7, Canada treatment approaches $[4,5,13]$. As recommended by recent head and neck cancer treatment guidelines, it is important to establish a pathologic diagnosis of HPV/ p16 OPSCC for purposes of prognostication [14]. Despite well-characterized clinical and histopathological differences, the treatment algorithm for HPV/p16 positive and negative OPSCC remains unchanged.

Positron emission tomography-computed tomography (PET-CT) is an important tool for the diagnosis and surveillance of OPSCC $[12,15,16]$. PET-CT utilizes glucose metabolism to identify tumor metabolic activity, which can accurately identify locoregional disease better than CT alone, particularly in smaller nodes $[17,18]$. Rates of cervical lymph node metastases in HPV positive OPSCC have been reported to be $60-76 \%$ at initial presentation [19]. Sensitivity and specificity of detecting cervical node metastases with PET-CT is high, ranging from $84-92 \%$ and $95-99 \%$, respectively [18]. HPV 
positive nodal metastases often present as a cystic or necrotic neck mass which may be less metabolically active on PET [20]. Additionally, inflammatory nodes can present as FDG avid lymphadenopathy and could represent false positives on PET imaging. Combining PET with CT allows for precise anatomic localization and further characterization of these potential metastatic lymph nodes.

The clinical role of PET-CT to localize primary tumors and metastatic disease has been well-established; but researchers are continuing to try to identify specific imaging biomarkers that may help diagnose, provide information about prognosis and guide treatments for patients with OPSCC. Maximum standard uptake value (SUVmax) is a quantitative measure of the highest FDG uptake within the region of interest. It has been hypothesized by others that a high SUVmax would be associated with a poor outcome, reflecting the increased proliferation of the tumor cells [21]. However HPV positive tumors, known to have favorable outcomes, are thought to have higher metabolic activity with increased proliferation and a predilection for early metastasis [22, 23]. Recent data from Kendi et al. [24] support the hypothesis that higher SUVmax is associated with HPV/p16 positivity. There is therefore conflicting evidence regarding the association between SUVmax and HPV/p16 positivity in OPSCC [21, 24-26]. To this end, we aimed to determine the correlation between nodal SUVmax and p16 status of OPSCC tumors using a cohort of patients treated with primary surgery.

\section{Methods}

The University of Alberta Research Ethics Board granted ethics approval for the study (Pro00016426).

\section{Patients}

Patients were identified using a prospectively collected database (Alberta Cancer Registry [27] (ACR)) of patients treated for OPSCC from January 2009 - December 2014 at a tertiary cancer treatment facility in Alberta, Canada. All adult patients treated with a primary surgical approach with a pre-treatment PET-CT and p16 status of the OPSCC were included within the cohort (Fig. 1). Patients were categorized as smokers using a previously defined cutoff of $\geq 10$ pack years of tobacco use [4]. Patients were excluded if they had received previous treatment for head and neck cancer or found to have an N0 neck on surgical pathology. Initial demographic information was obtained from the ACR and verified by retrospective review of patients' charts. HPV-status was obtained by immunohistochemistry staining for $\mathrm{p} 16$, a surrogate marker of oncogenic HPV, as previously reported using established standards [11, 13, 28, 29].

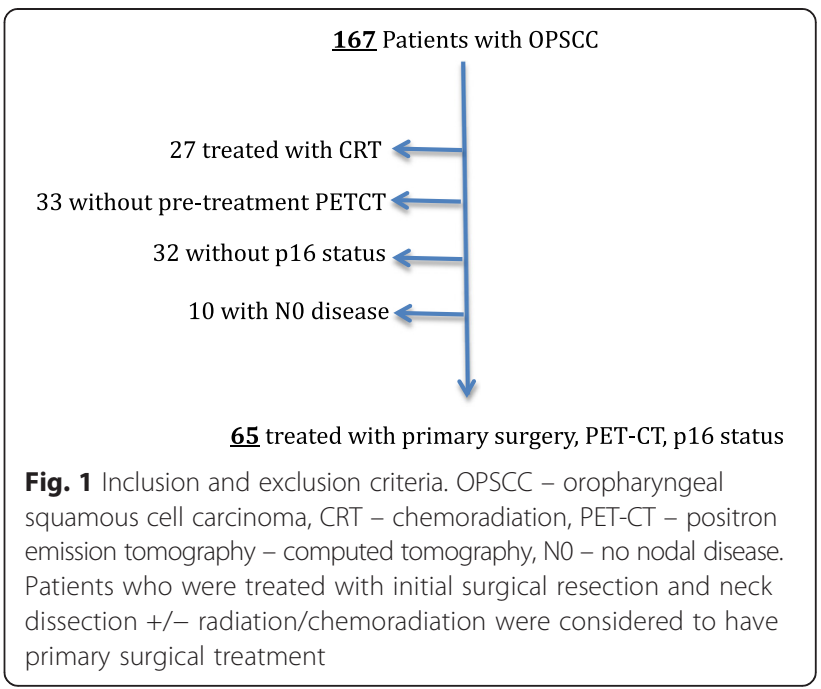

\section{PET-CT image analysis}

PET-CT protocols were obtained using two scanners, both Gemini TF 16-slice PET-CT scanner systems (Phillips Healthcare, Andover, MA). All patients were scanned with a consistent head and neck protocol. Following a fasting period of at least $4 \mathrm{~h}$ and serum baseline glucose measurements, patients were injected with $5.18 \mathrm{MBq} / \mathrm{kg}$ of 18 FDG tracer. A minimum of $60 \mathrm{~min}$ was waited following tracer injection and PET scanning. Two separate acquisitions were used to scan patients from skull vertex to mid thighs. Following the PET scan, patients were injected with intravenous contrast and a helical CT was performed to scan patients from skull vertex to mid thighs.

Using OASIS software (Segami Corporation, Columbus, Ohio), PET-CT images were reviewed by authors JC, CJ and TC. Reviewers were blinded to p16 status at the time of imaging review. Coronal, sagittal and transverse images were examined to identify SUVmax of the primary tumor and cervical nodal metastases. A spherical volumetric area of interest was focused upon individual lesions in the head and neck to identify metabolic parameters of the lesions. Final radiological and nuclear medicine reports were reviewed for additional correlation of findings. Only nodal and primary malignancies with FDG uptake were included within the analysis. The SUVmax for the primary as well as the nodal SUVmax were obtained from the OASIS software. In patients with multiple FDG avid cervical lymph nodes, the highest SUVmax was recorded, consistent with previous PET-CT protocols [25].

\section{Statistical analyses}

SPSS version 23.0 was used for statistical analyses (SPSS Inc., Chicago, IL, USA). Regression analyses of factors and covariates were performed to include age, gender, TNM staging, tumor subsite, treatment, smoking status, p16 positivity and SUVmax. ANOVA, Mann Whittney $U$ 
Table 1 Demographics and staging of oropharyngeal malignancies

\begin{tabular}{llll}
\hline Characteristic & P16+ & P16- & $P$ value \\
\hline Age (mean, SD) & $57.6,7.6$ & $59.5,10.6$ & 0.39 \\
Gender (\% male) & 88.2 & 76.9 & 0.06 \\
Smoking & 54.9 & 84.6 & $<0.001$ \\
Tumor stage & & & \\
T1 & 15.3 & 25.4 & 0.09 \\
T2 & 23.1 & 41.1 & \\
T3 & 15.3 & 15.7 & \\
T4 & 46.2 & 17.6 & \\
Nodal stage & & & 0.41 \\
N1 & 38.5 & 13.7 & \\
N2 & 53.8 & 80.4 & \\
N3 & 7.7 & 5.9 & \\
\hline
\end{tabular}

SD standard deviation

test, and the Kruskal-Wallis test were used to calculate differences between groups where appropriate. Statistical significance was defined as $\mathrm{p}<0.05$.

\section{Results}

A prospectively collected database identified 167 patients who were treated at the University of Alberta for OPSCC between 2009 and 2014. Of the patients treated with primary surgery, sixty-five had adequate pretreatment PET-CT and p16 status available (Fig. 1).

Patients with p16 negative disease were more likely to have a smoking history ( $>10$ pack years) (Table 1$)$. No statistically significant differences were seen between p16 positive and negative patients in terms of age at presentation, gender, $\mathrm{T}$ stage and $\mathrm{N}$ stage.

The mean SUV max of the primary tumor for p16 negative disease (13.7) was higher than that for $\mathrm{p} 16$ positive disease (10.3) but there was no significant difference when these were assessed with a Mann Whitney $U$ comparison of means $(p=0.28)$. The average nodal SUVmax for p16 positive disease (10.8) was significantly higher $(\mathrm{p}=0.02)$ than that for $\mathrm{p} 16$ negative disease (7.9) (Fig. 2).

Using the dependent variable, p16 status, a multinomial logistic regression analysis was performed (Table 2). The only variable that was found to be significant in predicting p16 status was nodal SUVmax. Higher nodal SUVmax was associated with p16 positive disease. Primary tumor SUVmax was not a significant predictor.

Using the mean SUVmax of p16 positive nodes as a significant predictor of p16 positive disease, we categorized patients according to this cutoff value (mean SUVmax p16+). Patients with nodal SUVmax $\geq$ mean SUVmax p16+ had a positive predictive value of $\mathrm{p} 16$ positive disease of $92.3 \%$. The diagnostic odds ratio (DOR) of the mean SUVmax p16+ was 4.9 (95 \% CI, 1.0-24.3)

\section{Discussion}

The emergence of PET-CT as a diagnostic tool for head and neck malignancies has allowed physicians to identify primary tumors and locoregional metastases early in the disease course. Many feel the role of PET-CT can be expanded to help characterize some of these lesions to more accurately diagnose and target therapy for different types of cancers. This study has shown a significant association between higher nodal SUVmax and p16 positive disease status. In addition, in patients who had a nodal SUVmax of greater than 10.8 (mean SUVmax p16+), we could reliably predict p16 positive disease $(\mathrm{DOR}=4.9)$. A biological basis for these findings could represent the higher cell division and oxygenation of p16 positive tumors or the immune response of HPV infected nodes, which could FDG activity [24].
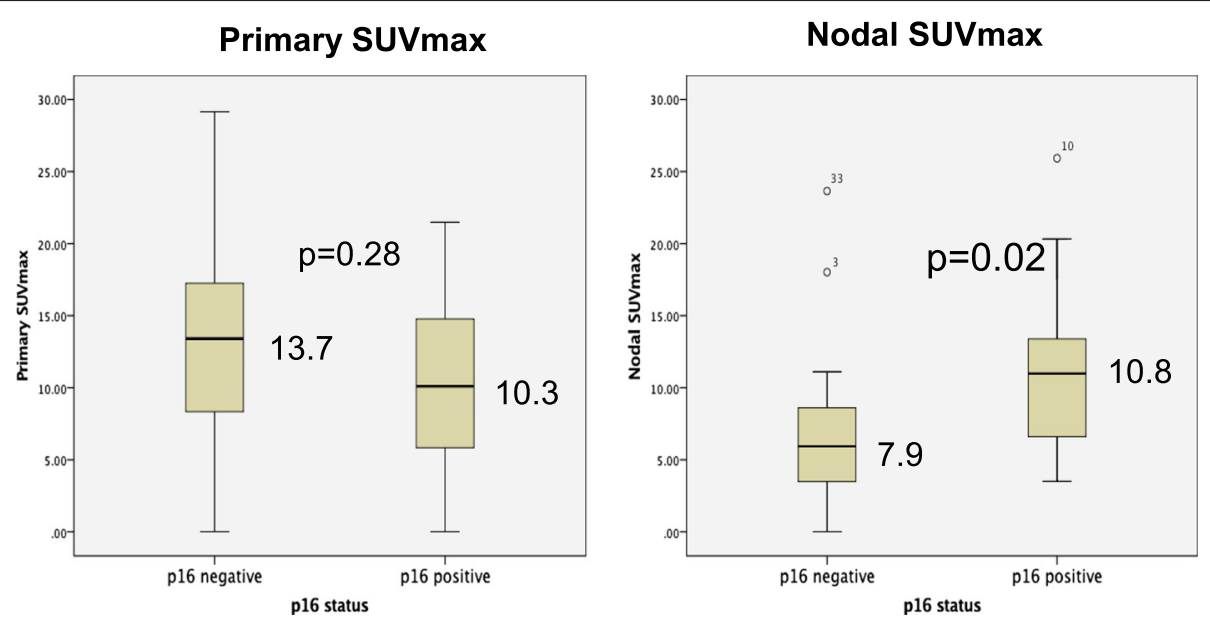

Fig. 2 Comparison of means for p16 positive and negative disease for primary and nodal maximum standard uptake value (SUVmax) 
Table 2 Multinomial analysis with p16 status as the dependent variable

\begin{tabular}{lcc}
\hline Covariate & Standardized coefficient & $P$ value \\
\hline Gender & -0.046 & 0.820 \\
T stage & 0.113 & 0.594 \\
N stage & 0.027 & 0.895 \\
Smoking & -0.075 & 0.712 \\
ECOG & -0.270 & 0.158 \\
ECS & 0.023 & 0.918 \\
Primary SUV max & -0.292 & 0.183 \\
Nodal SUVmax & 0.480 & 0.041 \\
\hline
\end{tabular}

ECOG Eastern Cooperative Oncology Group performance status, ECS extra-capsular spread, SUVmax maximum standard uptake value

Two similar studies examining the association of PET$\mathrm{CT}$ and HPV status have found conflicting results [24, 25]. Tahari and colleagues had a large cohort $(\mathrm{N}=123)$ and retrospectively examined the relationship between PETCT imaging markers and HPV status [25]. They found higher PET values in HPV positive nodal metastasis but this difference was not statistically significant. Kendi and colleagues [24] examined the relationship of PET-CT imaging markers and p16 status in patients with oral cavity and OPSCC . Consistent with results from our study, they found a statistically significantly higher nodal SUVmax, but not primary tumor SUVmax, in patients with HPV positive disease as compared to those with HPV negative cancer. Although their study had a relatively small sample size $(\mathrm{N}=22)$, they also identified a nodal SUVmax cut off value, which was predictive of HPV positivity (SUVmax $>7.66$ ). Our study supports a significant association between p16 status and nodal SUVmax. With this data, clinicians may be able to more accurately predict HPV status based on imaging in combination with other clinical parameters.

HPV positive tumors are being found in a new population - younger males without environmental risk factors such as tobacco and alcohol. Clinicians; however, may be slow to screen these individuals for malignancies, as they do not have the traditional exposures that have been historically associated with head and neck cancer. As a result, the average time for diagnosis of OPSCC is greater than 3 months from initial presentation at a general practioner's office [30]. Given the prognostic importance of p16 positivity in OPSCC, diagnostic information that can assist in triaging patients according to p16 status is valuable to both patients and physicians.

PET-CT also plays a role in identifying primary tumors in cases of cancer of unknown primary (CUP). Multiple studies have found HPV positive metastatic neck nodes are more likely to be associated with an oropharyngeal primary than other head and neck malignancies [31, 32]. Nodal SUVmax, identified on pre-treatment PET-CT could give additional information about the underlying malignancy for CUP. Having a high suspicion for p16 positive OPSCC based on imaging could help direct a more aggressive search for a CUP. Recent reports using transoral resection of base of tongue and tonsils in p16 positive CUPs have been $>90 \%$ effective in identifying the primary site $[33,34]$.

Our findings could have a particularly useful clinical implication in the diagnosis of OPSCC patients when surgical biopsy under general anesthesia may portend a high risk of morbidity. If imaging with PET-CT could help identify p16 status, clinicians may be able to tailor chemoradiation therapy in this population. It is thought the improved prognosis of HPV positive OPSCC relates in part to the increased radiosensitivity of the tumor. Currently HPV positive and negative tumors are treated equivocally. Given the variable pathologic and molecular behavior of HPV positive and negative tumors, targeted treatments may soon be developed that could allow for individualized therapies that may reduce overall radiation-related side effects and improve post-treatment quality of life.

This study has a number of limitations. Although initial data were collected prospectively, it remains a retrospective analysis in a single institution, which could limit the generalizability of the results. As shown in Fig. 1, several patients were excluded from the analysis due to lack of PET-CT or p16 status, which could bias our results. PET-CT protocols may vary between cancer treatment facilities, which may limit the extrapolation of nodal SUVmax cutoff values described herein. Finally, a relatively small patient cohort was used in this analysis. These limitations could be improved with further studies using prospective data from multiple institutions.

\section{Conclusions}

There is a significant association between p16 positivity and increased nodal SUVmax in OPSCC. Further larger scale studies involving other institutions would be recommended to examine the clinical utility of this association.

\section{Competing interests}

The authors declare that they have no competing interests.

\section{Authors' Contributions}

JC collected and analysed data, and drafted the manuscript. CJ, HZ and TC participated in data collection. DO, JH, HS and VB participated in data analysis and drafting the manuscript. All authors read and approved the final manuscript.

Received: 4 June 2015 Accepted: 2 September 2015

Published online: 15 September 2015

\section{References}

1. Hocking JS, Stein A, Conway EL, Regan D, Grulich A, Law M, et al. Head and neck cancer in Australia between 1982 and 2005 show increasing incidence of potentially HPV-associated oropharyngeal cancers. Br J Cancer. 2011;104:886-91.

2. Marur S, D'Souza G, Westra WH, Forastiere AA. HPV-associated head and neck cancer: a virus-related cancer epidemic. Lancet Oncol. 2010;11:781-9. 
3. Westra WH. The changing face of head and neck cancer in the 21st century: the impact of HPV on the epidemiology and pathology of oral cancer. Head Neck Pathol. 2009;3:78-81.

4. Ang KK, Harris J, Wheeler R, Weber R, Rosenthal DI, Nguyen-Tân PF, et al. Human papillomavirus and survival of patients with oropharyngeal cancer. N Engl J Med. 2010;363:24-35.

5. Gillison ML, Zhang Q, Jordan R, Xiao W, Westra WH, Trotti A, et al. Tobacco smoking and increased risk of death and progression for patients with p16-positive and p16-negative oropharyngeal cancer. J Clin Oncol. 2012;30:2102-11.

6. Andrews E, Seaman WT, Webster-Cyriaque J. Oropharyngeal carcinoma in non-smokers and non-drinkers: a role for HPV. Oral Oncol. 2009;45:486-91.

7. D'Souza G, Kreimer AR, Viscidi R, Pawlita M, Fakhry C, Koch WM, et al. Case-control study of human papillomavirus and oropharyngeal cancer. N Engl J Med. 2007;356:1944-56.

8. Kumar B, Cordell KG, Lee JS, Worden FP, Prince ME, Tran HH, et al. EGFR, p16, HPV Titer, BCl-xL and p53, sex, and smoking as indicators of response to therapy and survival in oropharyngeal cancer. J Clin Oncol. 2008;26:3128-37.

9. Biron VLV, Mohamed AA, Hendzel MJM, Underhill DDA, Seikaly HH. Epigenetic differences between human papillomavirus-positive and -negative oropharyngeal squamous cell carcinomas. J Otolaryngol Head Neck Surg. 2012;41 Suppl 1:S65-70.

10. van Kempen PM, Noorlag R, Braunius WW, Stegeman I, Willems SM, Grolman W. Differences in methylation profiles between HPV-positive and HPV-negative oropharynx squamous cell carcinoma: A systematic review. Epigenetics. 2014;9(2):194-203.

11. Cooper T, Biron V, Adam B, Klimowicz AC, Puttagunta L, Seikaly H. Prognostic utility of basaloid differentiation in oropharyngeal cancer. J Otolaryngol Head Neck Surg. 2013;42:57.

12. Xu CC, Biron VL, Puttagunta L, Seikaly H. HPV Status and second primary tumours in Oropharyngeal Squamous Cell Carcinoma. J Otolaryngol Head Neck Surg. 2013;42:36

13. Seikaly H, Biron VL, Zhang H, O'Connell DA, Côté DWJ, Ansari K et al. The role of primary surgery in the treatment of advanced oropharyngeal cancer. Head Neck 2015. doi:10.1002/hed.24042.

14. Pfister DG, Ang K-K, Brizel DM, Burtness BA, Busse PM, Caudell JJ, et al. Head and neck cancers, version 2.2013. Featured updates to the NCCN guidelines. J Natl Compr Canc Netw. 2013;11:917-23.

15. Pentenero M, Cistaro A, Brusa M, Ferraris MM, Pezzuto C, Carnino R, et al. Accuracy of 18 F-FDG-PET/CT for staging of oral squamous cell carcinoma. Head Neck. 2008;30:1488-96.

16. Kurien G, Hu J, Harris J, Seikaly H. Cost-effectiveness of positron emission tomography/computed tomography in the management of advanced head and neck cancer. J Otolaryngol Head Neck Surg. 2011;40:468-72.

17. Muylle K, Castaigne C, Flamen P. 18F-fluoro-2-deoxy-D-glucose positron emission tomographic imaging: recent developments in head and neck cancer. Curr Opin Oncol. 2005;17:249-53.

18. Yoon DY, Hwang HS, Chang SK, Rho Y-S, Ahn HY, Kim JH, et al. CT, MR, US,18F-FDG PET/CT, and their combined use for the assessment of cervical lymph node metastases in squamous cell carcinoma of the head and neck. Eur Radiol. 2009;19:634-42.

19. Lin DT, Cohen SM, Coppit GL, Burkey BB. Squamous cell carcinoma of the oropharynx and hypopharynx. Otolaryngol Clin North Am. 2005;38:59-74. viii.

20. Corey AS, Hudgins PA. Radiographic imaging of human papillomavirus related carcinomas of the oropharynx. Head Neck Pathol. 2012;6 Suppl 1:S25-40.

21. Hoshikawa H, Yamamoto Y, Mori T, Kishino T, Fukumura T, Samukawa Y, et al. Predictive value of SUV-based parameters derived from pre-treatment (18)F-FLT PET/CT for short-term outcome with head and neck cancers. Ann Nucl Med. 2014;28:1020-6.

22. Arenz A, Ziemann F, Mayer C, Wittig A, Dreffke K, Preising S, et al. Increased radiosensitivity of HPV-positive head and neck cancer cell lines due to cell cycle dysregulation and induction of apoptosis. Strahlenther Onkol. 2014;190:839-46.

23. Ziemann F, Arenz A, Preising S, Wittekindt C, Klussmann JP, EngenhartCabillic R, et al. Increased sensitivity of HPV-positive head and neck cancer cell lines to $\mathrm{x}$-irradiation \pm Cisplatin due to decreased expression of E6 and E7 oncoproteins and enhanced apoptosis. Am J Cancer Res. 2015;5:1017-31.

24. Kendi ATK, Magliocca K, Corey A, Nickleach DC, Galt J, Higgins K, et al. Do 18F-FDG PET/CT parameters in oropharyngeal and oral cavity squamous cell carcinomas indicate HPV status? Clin Nucl Med. 2015;40:e196-200.
25. Tahari AK, Alluri KC, Quon H, Koch W, Wahl RL, Subramaniam RM. FDG PET/CT imaging of oropharyngeal squamous cell carcinoma: characteristics of human papillomavirus-positive and -negative tumors. Clin Nucl Med. 2014;39:225-31.

26. Joo Y-H, Yoo I-R, Cho K-J, Park J-O, Nam I-C, Kim M-S. Preoperative 18F-FDG PET/CT and high-risk HPV in patients with oropharyngeal squamous cell carcinoma. Head Neck. 2014;36:323-7.

27. O Connell D, Seikaly H, Murphy R, Fung C, Cooper T, Knox A, et al. Primary surgery versus chemoradiotherapy for advanced oropharyngeal cancers: a longitudinal population study. J Otolaryngol Head Neck Surg. 2013;42:31.

28. Lau HY, Brar S, Klimowicz AC, Petrillo SK, Hao D, Brockton NT, et al. Prognostic significance of p16 in locally advanced squamous cell carcinoma of the head and neck treated with concurrent cisplatin and radiotherapy. Head Neck. 2011;33:251-6.

29. Begum S, Gillison ML, Ansari-Lari MA, Shah K, Westra WH. Detection of human papillomavirus in cervical lymph nodes: a highly effective strategy for localizing site of tumor origin. Clin Cancer Res. 2003;9:6469-75.

30. Ho T, Zahurak M, Koch WM. Prognostic significance of presentation-to-diagnosis interval in patients with oropharyngeal carcinoma. Arch Otolaryngol Head Neck Surg. 2004;130:45-51.

31. Fakhry C, Westra WH, Li S, Cmelak A, Ridge JA, Pinto H, et al. Improved survival of patients with human papillomavirus-positive head and neck squamous cell carcinoma in a prospective clinical trial. J Natl Cancer Inst. 2008;100:261-9.

32. Schmalbach CE, Miller FR. Occult primary head and neck carcinoma. Curr Oncol Rep. 2007;9:139-46.

33. Mehta $V$, Johnson $P$, Tassler A, Kim S, Ferris RL, Nance M, et al. A new paradigm for the diagnosis and management of unknown primary tumors of the head and neck: a role for transoral robotic surgery. Laryngoscope. 2013;123:146-51.

34. Karni RJ, Rich JT, Sinha P, Haughey BH. Transoral laser microsurgery: a new approach for unknown primaries of the head and neck. Laryngoscope. 2011;121:1194-201.

\section{Submit your next manuscript to BioMed Central and take full advantage of:}

- Convenient online submission

- Thorough peer review

- No space constraints or color figure charges

- Immediate publication on acceptance

- Inclusion in PubMed, CAS, Scopus and Google Scholar

- Research which is freely available for redistribution 